\begin{tabular}{|l|l|l|l|}
\hline Eiszeitalter und Gegenwart & Band 12 & Seite 92-98 & Ohringen/Württ. 1. November 1961 \\
\hline
\end{tabular}

\title{
Uber die Vegetationsentwicklung des Weichsel-Interstadials aus Hamburg-Bahrenfeld
}

\author{
Von R. Hallik und K. Kubitzki \\ (Mitteilung aus dem Geol. Landesamt Hamburg Nr. 40) \\ Mit 2 Abbildungen im Text.
}

\begin{abstract}
$\mathrm{Z}$ usammenfassung. Eine interstadiale, aus Gyttja und Torfen aufgebaute Lagefolge, die mit dem aus Dänemark und Holland beschriebenen Brörup-Interstadial parallelisiert wird, wurde po'lenanalytisch und z. T. hinsichtlich ihrer Makroflora untersucht. Danach ergibt sich der waldlose bis waldarme, durch Vorwalten von Zwergbirken und krautigen Pflanzen gekennzeichnete Abschnitt I und der Abschnitt II mit großer Bewaldungsdichte, in dem offenbar zunächst Birken und schließlich Kiefern und Fichten die Wälder beherrschten, während die lokale Moorvegetation ihr Gepräge durch das gleichzeitige Vorkommen von Zwergbirken und Bergkiefern erhielt, dessen pflanzengeographische Bedeutung gewürdigt wird.

$\mathrm{Su} \mathrm{m}$ m a r y. An interstadial deposit of mud and peat corresponding to the Danish and Dutch so called Brørup-interstadial has been investigated. A first series characterised by Betula nana and non arboreous vegetation as well as a second series of closed forests have been described. Birch, later on pine and spruce were the constituents of the forests.

The conception of the vegetation derived from polienanalytical investigation shows a considerable influence of the local vegetation on the fen with Betula nana and Pinus montana var. uncinata, to which particular attention has been paid.
\end{abstract}

\section{Ein le it ung}

Im Rahmen einer Serie von Untersuchungsbohrungen, die das Geologische Landesamt Hamburg im Jahre 1959 niederbringen ließ, wurde bei Bahrenfeld ein Torf-Flöz angefahren, dessen stratigraphische Stellung zunächst unklar erschien. Die nur lückenhaft entnommenen Proben ließen lediglich eine Gleichaltrigkeit mit dem FrühweichselInterstadial von Harksheide (HALLIK 1955) feststellen. Erst während einer Wiederholung der Bohrung im Jahr 1960 konnte ein geschlossenes Profil entnommen werden. Es schien berechtigt zu sein, die Vegetationsentwicklung dieses erst durch wenige Fundpunkte bekannt gewordenen Interstadials in Nordwestdeutschland im Einzelnen zu untersuchen.

Der hier bearbeitete Fundpunkt liegt ca. 200 m nördlich der Bahnlinie Altona-Blankenese, ca. 0,5 km westlich des Bahnhofs Bahrenfeld. An dieser Stelle befindet sich eines von den zahlreichen Senkungsgebieten über dem Salzstock von Langenfelde. Es ist mit großer Wahrscheinlichkeit anzunehmen, daß einzelne Erdfälle des Senkungsgebietes während der Endphase des Eem (Ein Weichsel-Frühglazial, Hallik 1952) entstanden wären. Die Erdfälle sind, soweit bisher bekannt, mit z. T. lehmig-kiesigen Sanden, die als Fließcrden anzusehen sind, gefüllt. In einigen von diesen sind bis zu ca. $1 \mathrm{~m}$ mächtige organogene Horizonte eingeschaltet. Im ganzen Senkungsgebiet sind holozäne Torfe von wechselnder Mächtigkeit weit verbreitet.

\section{Lagerungsverhäl $\mathrm{t} n$ is se}

Die beigefügte Skizze (Abb. 1), die auf Grund einer älteren Serie von Bohrungen aus diesem Gebiet konstruiert ist, zeigt die Lagerungsverhältnisse, soweit sie bisher bekannt geworden sind.

Die Altersstellung ist bereits kurz erwähnt worden. Über die Vergleichsmöglichkeiten mit anderen Lokalitäten soll in einem anderen Abschnitt dieser Mitteilung berichtet werden. 


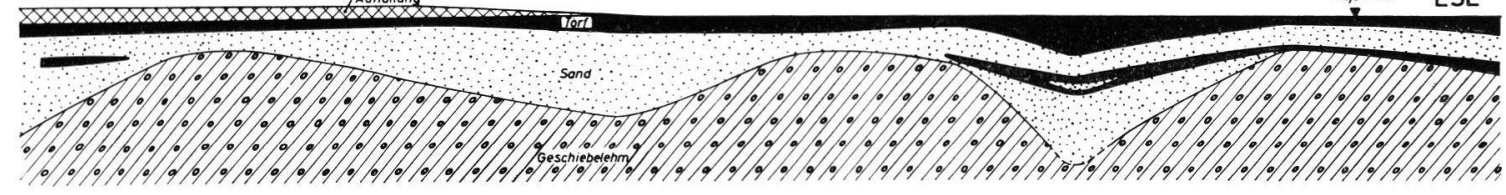

Abb. 1. Lagerungsverhältnisse der organogenen Serien des Weichsel-Interstadials und des Holozäns in Hamburg-Bahrenfeld.

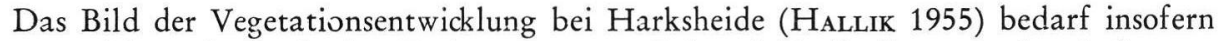
einer Revision, als durch das Vorkommen von Bahrenfeld die Deutung des Auftretens thermophiler Elemente im Harksheider Diagramm teilweise abzuändern wäre.

Das bearbeitete Profil hat folgenden Aufbau:
$0-2,40 \mathrm{~m}$
Aufschüttung
$2,40-4,00 \mathrm{~m}$
$4,00-5,00 \mathrm{~m}$
Flachmoortorfe des Holozäns, trockenere und nassere Lagen wechselnd.
$5,00-6,95 \mathrm{~m}$
$6,95-7,26 \mathrm{~m}$
Sand, mittel - fein.
Sand, fein - mittel, mit Kieseleinlagerungen.
$7,26-7,33 \mathrm{~m}$
mesotropher Sphagnum-Torf mit Resten von Pinus mugo, im unteren
Teil Betula nana.
$7,33-7,48 \mathrm{~m}$
stark zersetzter mesotropher Sphagnum-Torf mit Betula nana. Viel-
leicht schwingrasenartige Bildung.
$7,48-7,67 \mathrm{~m}$
Grobdetritus-Gyttja.
$7,67-8,11 \mathrm{~m}$
schluffige Grobdetritus-Gyttja. Im oberen Teil reichlich Nuphar, im unteren Tei! Myriophyllum alterniflorum.
8,11-8,50 m Sand. (Bohrung abgebrochen wegen technischem Unfall.)
sandige Fein- bis Grobdetritus- Gyttja. Im oberen Teil reich'ich Myrio- phylium alterniflorum.

\section{Die Vegetationsentwicklung (Abb. 2)}

Entsprechend dem üblichen Vorgehen wird die Vegetationsentwicklung hier so gegliedert, daß sich Abschnitte von einheitlichem floristischem Gepräge ergeben. So steht Abschnitt I als waldarme oder waldlose Phase dem waldreichen Abschnitt II gegenüber. Mit der gleichen Berechtigung könnte die Grenze zwischen beiden wie die Begrenzung der Unterabschnitte aber auch an jene Punkte gelegt werden, an denen ein Umschwung in der Vegetationsentwicklung erfolgt; die wesentlichste Zäsur wäre dann in das Niveau von Spektrum $15 \mathrm{zu}$ legen, in dem der Rückgang der Nichtbaumpollen (NBP) beginnt und sich damit eine Ausbreitung der Wälder anbahnt. Eine solche Gliederung würde durch das Bestreben gerechtfertigt werden, aus dem Beginn der Kurvenausschläge die Ursachen für die Änderungen in der Vegetation zu erfassen und damit über größere Entfernungen synchrone Niveaus zu erhalten.

A bschnitt I zeichnet sich, wie erwähnt, durch hohe NBP-Werte aus; der BetuiaPollen gehört mit 5-15\%, meist mit weniger als 10\% der Betula-Pollensumme nach Größe und Gestalt dem B. nana-Typ an; eine quantitative Trennung war jedoch der schlechten Pollenerhaltung wegen weder größenstatistisch noch morphologisch möglich. Die schlechte Pollenerhaltung ist auch der Grund dafür, daß der Pollen von Potamogeton, Populus und Juniperus nicht mehr bestimmbar war. Der Pollen von Picea und von den wärmebedürftigen Gehölzen, z. T. auch von Pinus, dürfte in diesem Abschnitt größtenteils sekundärer Herkunft sein; eine Subtraktion wurde jedoch nicht vorgenommen. Der Florencharakter zeigt weitgehende Übereinstimmung zu dem der spätglazialen Flora, wie sie in den letzten Jahrzehnten in Europa nördlich der Alpen in so überraschend gleichmäßiger Ausprägung bekannt geworden ist; ihre Reste leben heute an waldlosen Standorten: teils am Meeresstrand (Plantago maritima, Arten von Armeria und Centaurium), teils im Gefolge des Menschen als Unkräuter (Plantago major und media, Polygonatum aviculart) und teils in Trockenrasen. 


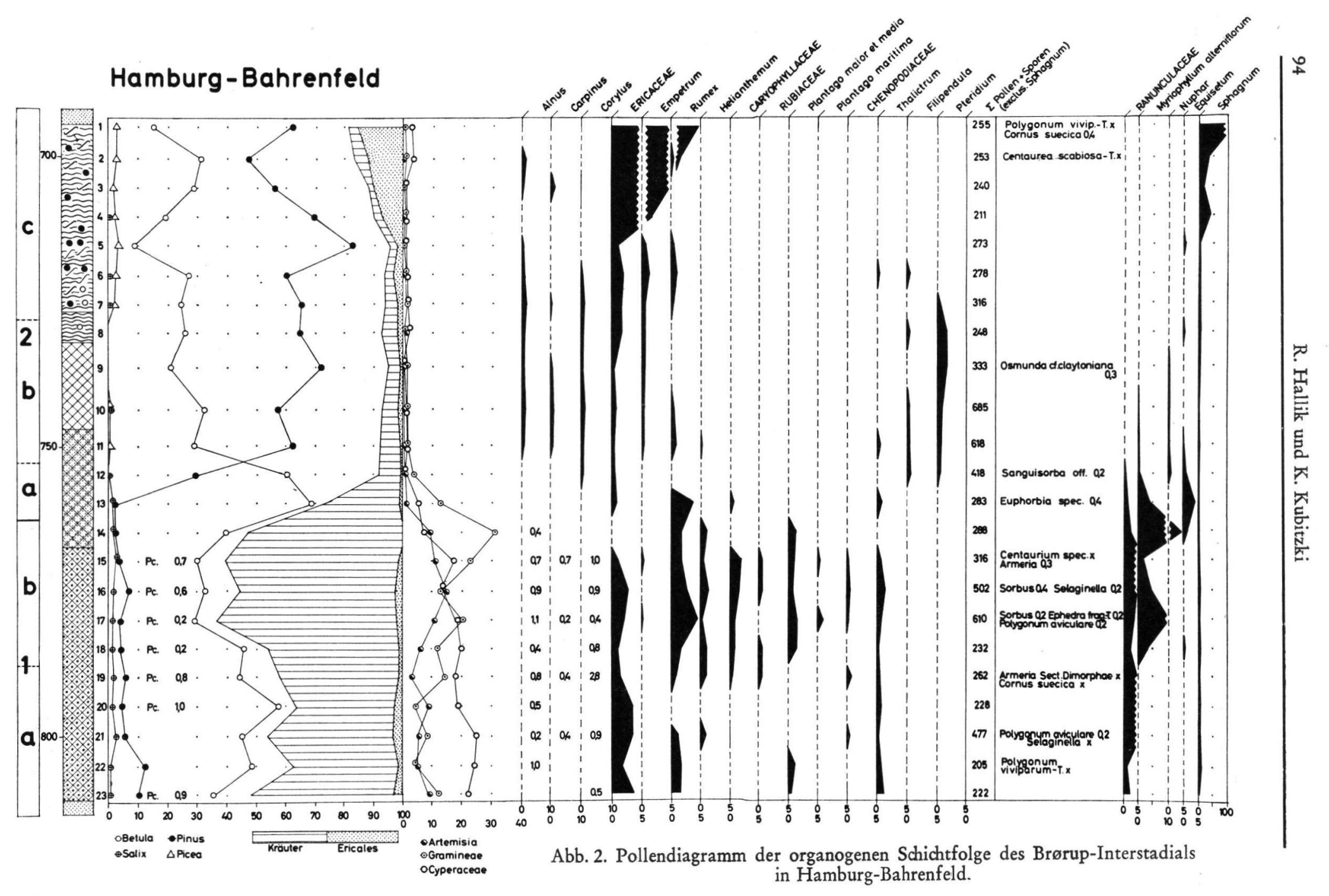


Von Armeria wurde Pollen vom A- und B-Typ i. S. von Iversen (1940) gefunden; es handelt sich also um eine Art der Sektion Dimorphae (IVERSEN), und zwar, so weit das nach der von Szafer (1945) gegebenen Pollenbeschreibung zu sagen ist, offenbar nicht um Armeria iverseni, sondern um A.maritima oder um eine ihr nahestehende Art. BAKER (1959) hat darauf hingewiesen, daß schon geringfügige Beschattung das Blühen von Armeria maritima hintanhält. Das Vorkommen des Rutenstrauches Ephedra (1 Pollenkorn, E. fragilis-Typ ss. WeLTEN (1957), $65 \times 15 \mu$, mit ca. 8 Falten) überrascht nicht mehr, da diese Gattung nicht nur im Spät- und z. T. noch im Postglazial, sondern nach GRITSchuK (1955) auch in älteren Abschnitten des Pleistozäns in Europa weit verbreitet war.

Die Untergliederung des Abschnittes I grïndet sich auf das Verhältnis der $\Sigma$ NBP zum Betula-Pollen. Der Anstieg der NBP an der Grenze Ia/b geht vor allem auf Pflanzen zurück, die mit mehr oder weniger großer Wahrscheinlichkeit für trockene Standorte bezeichnend sind (Artemisia, Rumex cf. acetosella, Helianthemum u. a.). Die Ursache für die Zunahme der Kräuterpollen ist schwer anzugeben; in Anbetracht des gleichzeitigen Anstieges der Myriophyllum alterniflorum-Kurve könnte man an eine Temperaturzunahme und im Zusammenhang damit an eine Ausweitung der trockenen Standorte denken.

Abschnitt II : Schon gegen Ende des Abschnittes Ib ist Betula stark gefördert, während die NBP zurückgehen; lediglich die Gramineen kommen vorübergehend zu großer Häufigkeit. In Spektrum 14 stammt ungefähr noch die Hälfte des Betula-Pollens von der Zwergbirke, die aber in den darüberfolgenden Spektren fast ganz von Baumbirken abgelöst wird, obwohl der Torf bis $720 \mathrm{~cm}$ Betula nana-Blättchen enthält. In der Krautvegetation verschwinden die Heliophyten, und Filipendula tritt häufiger auf, wie dies vom Beginn des Postglazials bekannt ist. Der gleichzeitige Anstieg der PteridiumKurve ist dagegen ein wichtiger Unterschied zum Postglazial: denn in Norddeutschland und Dänemark fehlt Pteridium im Präboreal und frühen Boreal bis zum Anstieg der Eichenmischwald-Kurve (vgl. z. B. Diagramme von Jørgensen 1954 und Kubitzki 1961). Das Verhalten von Pteridium im Postglazial und seine heutige Verbreitung in N-Europa (geschlossenes Verbreitungsgebiet in Finnland und Schweden bis $63^{\circ} \mathrm{n}$. Br., in Norwegen bis zum Wendekreis) könnte also ein Hinweis auf günstigere Temperaturverhältnisse zu Beginn der Zone II sein.

In der Zeit der größten Häufigkeit der Baumbirke beginnt schlagartig die Ausbreitung von Pinus. Der rationelle Anstieg der Picea-Kurve ist Veranlassung zur Abgrenzung der Abschnitte IIb und c. Da bei $735 \mathrm{~cm}$ die limnische Phase von sedentärer Torfbildung abgelöst wird, kann der Picea-Pollen nicht sekundärer Herkunft sein; und daß es sich um ferntransportierten Pollen handelt, wie in einer früheren Veröffentlichung angenommen (HaLliK 1955, S. 122), ist nicht wahrscheinlich, da die Pollenproduktion der lokalen Kiefern- und Birkenbestände den Fernfluganteil sicher stark unterdrückt haben dürfte. Auf die Frage nach der Deutung der Pollenwerte der übrigen wärmebedürftigen Holzarten, die in einem ähnlichen Falle schon einmal von SELLE (1954, S. 179) gestellt worden ist, möchten wir hier nicht eingehen. Vielmehr sei erwähnt, daß im Reisertorf von $725-700 \mathrm{~cm}$ reichlich Kurztriebe und Nadeln von Pinus mugo gefunden wurden, die nach Gestalt der Epidermiszellen sicher bestimmbar waren; bei $716 \mathrm{~cm}$ kamen auch zwei Zapfen von Pinus mugo cf. var. uncinata vor. Diese Art ist aus Norddeutschland sowohl aus dem HolsteinInterglazial (Ütersen-Glinde, vgl. H. SChroeder \& J. Stoller 1907) und dem Ausklang eines Interglaziales bei Lüneburg (G. MüLLER \& C. A. WEBER 1904) bekannt, das anläßlich einer Nachuntersuchung (HALLIK 1952) ins Eem-Interglazial gestellt wurde. Im Hinblick hierauf muß das heutige Fehlen von Pinus mugo in Nordeuropa befremden, wenngleich ihr einstiges Vorkommen in Norddeutschland das gleichzeitige Vorhandensein in Skandinavien keineswegs einschließt. Daß in Bahrenfeld neben Pinus mugo auch Pinus silvestris vertreten war, ist nicht durch Großreste bewiesen, aber in Anbetracht der aus 
dem Vorkommen von Picea und besonders Pteridium ableitbaren günstigen Temperaturverhältnisse durchaus möglich. Übrigens ist Pinus silvestris durch Makroreste aus dem Würm-Interstadial von Chelford (England; vgl. J. M. Simpson \& R. G. West 1959) und dem Interstadial in der Grube Marga bei Senftenberg/Lausitz belegt (Firbas \& GrahmanN 1928), das in seiner Vegetationsentwicklung Anklänge an Bahrenfeld zeigt und auch schon - allerdings abweichend von der Auffassung Grahmanns - von H. Gams (1935) in die Würmeiszeit gestellt worden ist. Jedenfalls dürfte das lokale Kiefernvorkommen in der Vegetation des Bahrenfelder Moores die Ursache für die hier im Gegensatz zum Diagramm Harksheide (HALLIK 1955) besonders niedrigen NBP-Werte sein, die deshalb eine hohe Bewaldungsdichte vielleicht nur vortäuschen.

Das Lüneburger Interglazial ist weiterhin durch das Vorkommen von Picea omorikoides ausgezeichnet, die nach WEBER als „stärker ausgeprägt alpine Form" der Picea omorika anzusehen ist. So war $P$. omorikoides auch in unserem Interstadial zu erwarten; doch ergab ein Vergleich mit rezenten Pollen, daß Picea hier dem P. abies-Typ angehört. In dem schon erwähnten Interstadial von Chelford fanden sich sogar Zavfen von Picea abies. Nach zahlreichen sorgfältigen Untersuchungen aus Polen (vgl. Szafer 1953) ist dort Picea omorikoides nur bis zum vorletzten. Interglazial vorgekommen, jedoch im letzten Interglazial nicht mehr gefunden worden. Auch Jessen \& Milthers (1928, S. 373) heben das Fehlen von Picea omorikoides (und übrigens auch von Pinus mugo) in Dänemark während des letzten Interglazials hervor. So bliebe Lüneburg der einzige Fundort jener Art aus dem Eem-Interglazial in Norddeutschland; die zeitliche Einstufung dieses Vorkommens sollte deshalb noch einmal überprüft werden.

Anders liegen die Dinge im Alpenvorland, wo Pollen von Picea cf. omorikoides von H. Reich (1953, S. 416 ff) wohl noch im 1. Würm-Interstadial gefunden worden ist (vgl. auch LüDi 1953). Offenbar sind also die an subarktische Verhältnisse angepaßten Biotypen von Picea omorika, zu denen wohl P.omorikoides zu rechnen ist, im Laufe des Pleistozäns nach und nach erloschen, und zwar im Norden anscheinend eher als am nördlichen Alpenrand. Sie dürften jedenfalls geringere Temperaturansprüche gehabt haben als die heute reliktisch in Jugoslawien vorkommende Picea omorika. Es sei erwähnt, daß H. GAMS (1954) immerhin mit der Möglichkeit rechnet, daß unter den vielen Formen unserer heutigen Fichte Kreuzungsprodukte mit Picea omorika weiterleben, so z. B. in Picea abies var. fennica REgeL. Schließlich sei hier der Fund einer Spore von Osmunda cf. claytoniana im Abschnitt IIb erwähnt, weil er den Nachweis bringt, daß diese heute vor allem in Fichtenwäldern Nordamerikas und Ostasiens lebende Art bis in die Würm-Eiszeit in der Holarktis viel weiter verbreitet war. $\left.{ }^{1}\right)$ Sporenfunde von Osmunda claytoniana aus dem Günz-Mindel- und Mindel-Riß-Interglazial aus Polen hat Szafer (1953) zusammengestellt. In diesem Zusammenhang sei darauf hingewiesen, daß bei der Bearbeitung pleistozäner Floren die Bestimmung der Taxa bis $\mathrm{zur}$ A rt eine ebenso dringliche wie lohnende Aufgabe darstellt, weil auf diesem Wege einerseits interessante florengeschichtliche Zusammenhänge aufgedeckt werden können, andererseits das ök olog is che Verständnis der Vegetationsgeschichte der weiter zurückliegenden Abschnitte des Pleistozäns, das uns augenblicklich noch fast vollständig abgeht, die Kenntnis der beteiligt gewesenen A r te $\mathrm{n}$ und nicht nur der Gattungen voraussetzt.

Der Abschnitt IIc ist im übrigen noch durch einen Anstieg der Ericaceen-Kurve ausgezeichnet, der allerdings vielleicht allein auf die Moorvegetation zurückgeht, da die im gleichen Niveau stark ansteigende Sphagnum-Kurve einen Umschwung der Moorentwicklung anzeigt. Die Ursache dieses Umschwunges dürfte am ehesten eine Feuchtigkeitszunahme und Abkühlung sein, so daß man hierin den ersten Ausdruck einer Revertenz der

1) Herrn Prof. Dr. Gunnar EndtMan sei bestens dafür gedankt, daß er 1955 Osmunda claytonia-Sporen aus dem Interstadial Waldfrieden (HALLIK 1955) bestimmt und damit unser Interesse auf diese Art gelenkt hat. 
Klimaentwicklung sehen kann. Sicher auf einer beginnenden Auflichtung der Wälder beruht wohl das regelmäßige Auftreten von Cornus suecica in diesem Abschnitt, das in einem unveröffentlichten Diagramm von Bahrenfeld und dem Diagramm Harksheide (HALlik 1955, Abb. 3) zum Ausdruck kommt, wo dieser Pollen als „x-Pollen“ bezeichnet wurde. Identisch damit ist übrigens nach einer Überprüfung auch der bei Hallik (1952) als „Rhamnus frangula?" angegebene Pollen.

In diesem Rahmen soll auf die reiche, in den letzten Jahren herangewachsene Literatur zur Frage der Gliederung der letzten Eiszeit nicht eingegangen werden. Nur die Beziehungen zu dem von Sv. Th. Andersen (1958; vgl. auch Andersen, de Vries \& Z Zagwijn 1960) aus Jütland beschriebenen Brörup-Interstadial sollen hier kurz erörtert werden. Während an der Gleichsetzung jenes Interstadials mit dem hier beschriebenen Vorkommen nicht gezweifelt werden kann, ist doch eine Parallelisierung im Einzelnen nicht möglich. Andersen (1958, S. 184)hat darauf hingewiesen, daß die Einwanderung der Kiefer und der Fichte offenbar nicht klimatisch bedingt war, sondern mit einer gewissen Verzögerung erfolgte. Schon daraus kann in verschiedenen Gebieten ein unterschiedlicher Verlauf der Waldgeschichte resultieren. So ist es nicht zu sagen, ob unserem Abschnitt II a bei ANDERSEN der ganze Abschnitt W 3 entspricht oder nur e in e r der vorübergehenden Birkenvorstöße in Jütland, nämlich W 3 a, c oder d. Ebenso fraglich ist es, ob die Zeit der hohen NBP-Werte, I b, mit W 3 b oder d oder mit der 2 . Hälfte von W 2 gleichgesetzt werden kann. Wir neigen zu letzter Möglichkeit, weil in Abschnitt I der Baumbirkenpollen in Bahrenfeld selten ist, was auch für W 1 und W 2 a und b in Jütland gilt, während in W 3 zumindest zeitweise Baumbirken schon verbreitet waren, die in Bahrenfeld erst gegen Ende von I b häufiger wurden. Danach müßte in Bahrenfeld gleichzeitig mit dem BrörupInterstadial das sog. Rodebaek-Interstadial erf̂ß sein, wogegen aber wieder einzuwenden ist, daß für Ia die Annahme höherer Temperaturen als I b im Augenblick nicht zu beweisen und nach unserer Deutung des Diagrammes sogar recht unwahrscheinlich ist. Wir möchten deshalb von einer Parallelisierung im Einzelnen der beiden Diagramme absehen.

\section{S chriften}

Andersen, Sv. Th.: New investigations of interglacial freshwater deposits in Jutland. - Eiszeitalter und Gegenwart, 8, S. 181-186, 1957.

Andersen, Sv. Th., De Vries, Hl. \& Z Z Weichselian glacial of Denmark and the Netherlands. - Geologie en Mijnbouw, 39, S. $38-42,1960$.

BAKER, H. G.: The contribution of autecological and geneco'ogical studies to our knowledge of the past migrations of plants. - The American Naturalist, 93, S. 255-272, 1959.

Firbas, F. \& Grahmann, R.: Uber jungdiluviale und alluviale Torflager in der Grube Marga bei Senftenberg (Niederlausitz). - Abh. math.-phys. Klasse d. Sächs. Akad. d. Wiss., 40, $4,1928$.

Gams, H.: Beiträge zur Mikrostratigraphie und Paläontologie des Pliozäns und Pleistozäns in Mittel- und Osteuropa und Westsibirien. - Eclogae geologicae Helvetiae, 28, S. 1-31, 1935. - - Das Verschwinden von Gehölzen aus den Alpen während des Eiszeitalters. Festschr. f. E. Aichinger, 1, Sonderfolge d. Schriftr. angew. Pflanzensoziologie, S. 71-76, 1954.

Hallik, R.: Ein „Weichsel-Frühglazial“-Profil in Lüneburg. - Eiszeitalter und Gegenwart, 2, S. 168-172, 1952. - - Uber eine Verlandungsfolge weichsel-interstadialen Alters in Harksheide bei Hamburg. - Ebenda, 6, S. 116-124, 1955.

Iversen, J.: B ütenbiologische Studien I. - Dimorphie und Monomorphie bei Armeria. - Det Kgl. Danske Vidensk. Selskab, Biolog. Meddel., 15, 8, 1940.

Jessen, K. \& Milthers, V.: Stratigraphical and paleontological studies of interglacial fresh-water deposits. - Danm. geol. Unders., II. R., Nr. 40, 1928.

Jørgensen, Sv.: A pollen analytical dating of Maglemose finds in the bog Aamosen, Zealand. Ebenda, II. R., Nr. 80, S. 159-187, 1954.

Kubitzki, K.: Zur Synchronisierung der nordwesteuropäischen Pollendiagramme. - Flora, 150, S. $43-72,1961$. 
LÜDI, W.: Die Pflanzenwelt des Eiszeitalters im nördlichen Vorland der Schweizer Alpen. - Veröff. geobotan. Inst. Rübel, Zürich, 27, 1953.

MÜLLER, G. \& WEBER, C. A.: Ưber eine frühdiluviale und vorglaziale Flora bei Lüneburg. - Abh. Kgl. Preuß. geol. Landesanstalt, N. F., 40, 1904.

REICH, H.: Die Vegetationsentwicklung der Interglaziale von Großweil-Ohlstadt und Pfefferbichl im Bayrischen Alpenvorland. - Flora, 140, S. 386-443, 1953.

Schroeder, H. \& Stoller, J.: Diluviale marine und Süßwasserschichten bei Uetersen-Schulau. Jb. d. Kgl. Preuß. geol. Landesanst. u. Bergakad. f. 1906, 27, 1907.

SELLE, W.: Das Vechelder Interstadial - Eiszeitalter und Gegenwart, 4/5, S. 176-180, 1954.

SimpSON, J. M. \& WEST, R. G.: On the stratigraphy and paleobotany of a late-pleistocene organic deposit at Chelford, Cheshire. - The new Phytologist, 57, S. 239-250, 1958.

Szafer, W.: The fossil Armeria in the European pleistocene, especially in Poland. - Starunia, 20, 1945 (polnisch $\mathrm{m}$. deutscher Zusf.). - - Pleistocene stratigraphy of Poland from the floristical point of view. - Rocznik Polskiego Towarzystwo Geologicznego, 22, S. 1-99, 1953. (Polnisch $\mathrm{m}$. engl. Zusf.).

Manuskr. eingeg. 26. 7. 1961.

Anschrift der Verf.: Dr. Rudolf Hallik, Hamburg, Rothenbaumchaussee 64a, Geolog. Landesamt; Dr. Klaus Kubitzki, Instituto de Botanica, Casilla 1094, Valdivia/Chile. 